\title{
Collisional Processes in Supersymmetric Plasma
}

\author{
Alina Czajka \\ Institute of Physics, Jan Kochanowski University, 25-406 Kielce, Poland \\ Stanisław Mrówczyński \\ Institute of Physics, Jan Kochanowski University, 25-406 Kielce, Poland and \\ National Centre for Nuclear Research, 00-681 Warsaw, Poland
}

(Dated: October 15, 2011)

\begin{abstract}
Collisional processes in ultrarelativistic $\mathcal{N}=1$ SUSY QED plasma are studied and compared to those in an electromagnetic plasma of electrons, positrons and photons. Cross sections of all binary interactions which occur in the supersymmetric plasma at the order of $e^{4}$ are computed. Some processes, in particular the Compton scattering on selectrons, appear to be independent of momentum transfer and thus they are qualitatively different from processes in an electromagnetic plasma. It suggests that transport properties of the SUSY plasma are different than those of its nonsupersymmetric counterpart. Energy loss and momentum broadening of a particle traversing the supersymmetric plasma are discussed in detail and the characteristics are shown to be surprisingly similar to those of QED plasma.
\end{abstract}

\section{INTRODUCTION}

This is our second paper where the ultrarelativistic $\mathcal{N}=1$ SUSY QED plasma is studied and compared to an electromagnetic plasma of electrons, positrons and photons. In the first one [1] we analyzed collective excitations of a supersymmetric plasma finding a rather surprising similarity between the two systems. Here we focus on collisional processes which control transport properties of the plasmas.

Our motivation to study the supersymmetric plasma is twofold. First of all supersymmetry is a good candidate to be a symmetry of Nature at sufficiently high energies and if true, supersymmetric plasmas existed in the early Universe. Experiments at the Large Hadron Collider can soon show whether there is any evidence to support the idea of supersymmetry. However, independently of its ontological status, supersymmetric field theories are worth studying because of their unique features. The AdS/CFT duality of the five-dimensional gravity in the anti de Sitter geometry and the conformal field theories, see the review [2], revived a great interest in the $\mathcal{N}=4$ supersymmetric Yang-Mills theory which is both classically and quantum mechanically conformally invariant.

AdS/CFT duality has provided a method to study strongly coupled field theories and numerous interesting results have been obtained, see the reviews 3, 4, but the relevance of the results for non-supersymmetric theories, which are of actual interest, is somewhat unclear. A systematic comparative analyses of supersymmetric systems and their nonsupersymmetric counterparts can be done in the domain of weak coupling where perturbative methods are applicable and several studies have been performed [5-10. We are particularly interested in non-equilibrium plasmas, which have not attracted much attention yet. We have started with the supersymmetric $\mathcal{N}=1$ QED plasma which is noticeably simpler than that of $\mathcal{N}=4$ Super Yang-Mills.

As already mentioned, we discuss here collisional processes in the $\mathcal{N}=1$ QED plasma which is assumed to be ultrarelativistic and thus all particles are treated as massless. We first compute cross sections of all binary processes which occur at the lowest nontrivial order of $\alpha \equiv e^{2} / 4 \pi$. There appears to be a class of binary interactions in the SUSY plasma which are qualitatively different than those in the electromagnetic plasma of electrons, positrons and photons where the interactions with small momentum transfer are dominant. For example, Compton scattering on selectrons is isotropic in the center-of-mass frame while the usual Compton scattering on electrons mostly occurs at small angles. Since collisional processes determine transport properties of a many-body system, the supersymmetric plasma can be expected to be rather different than its non-supersymmetric counterpart. It should be remembered, however, that the temperature is the only dimensional parameter which characterizes the equilibrium ultrarelativistic plasma. Consequently, the parametric form of transport coefficients can be determined by dimensional arguments. For example, the shear viscosity must be proportional to $T^{3} / \alpha^{2}$ and it is thus hard to expect that the viscosity of supersymmetric plasma is qualitatively different than that of electromagnetic one. Indeed, the shear viscosity of an $\mathcal{N}=4$ Super Yang Mills plasma is rather similar that of a quark-gluon plasma [6].

We consider here two transport characteristics of the $\mathcal{N}=1$ QED plasma which are not so constrained by dimensional arguments. Specifically, we compute the collisional energy loss and momentum broadening of a particle traversing the equilibrium plasma. The latter quantity determines a magnitude of radiative energy loss of highly energetic particle in a plasma [11. The dimensional argument does not work here because the two quantities depend not only on the plasma temperature but on the energy of test particle as well. We show that the energy loss and 
momentum broadening in SUSY plasma appear to be surprisingly similar to those in electromagnetic one.

Our paper is organized as follows. In the subsequent section, we introduce $\mathcal{N}=1$ SUSY QED by writing down and discussing its lagrangian. In Sec. III we enlist and analyze the binary processes which occur in the plasma. Secs. IV and $\mathrm{V}$ are devoted to the problem of, respectively, energy loss and momentum broadening of a test particle. The paper is closed with a summary of our main results and conclusions. The natural system of units with $c=\hbar=k_{B}=1$ and the signature of the metric tensor (+- - ) are used throughout the article.

\section{II. $\mathcal{N}=1$ SUSY QED}

The lagrangian of $\mathcal{N}=1$ SUSY QED is known, see e.g. [12, to be

$$
\begin{aligned}
\mathcal{L}= & -\frac{1}{4} F^{\mu \nu} F_{\mu \nu}+i \bar{\Psi} \not D \Psi+\frac{i}{2} \bar{\Lambda} \not \supset \Lambda+\left(D_{\mu} \phi_{L}\right)^{*}\left(D^{\mu} \phi_{L}\right)+\left(D_{\mu}^{*} \phi_{R}\right)\left(D^{\mu} \phi_{R}^{*}\right) \\
& +\sqrt{2} e\left(\bar{\Psi} P_{R} \Lambda \phi_{L}-\bar{\Psi} P_{L} \Lambda \phi_{R}^{*}+\phi_{L}^{*} \bar{\Lambda} P_{L} \Psi-\phi_{R} \bar{\Lambda} P_{R} \Psi\right)-\frac{e^{2}}{2}\left(\phi_{L}^{*} \phi_{L}-\phi_{R}^{*} \phi_{R}\right)^{2},
\end{aligned}
$$

where the strength tensor $F^{\mu \nu}$ is expressed through the electromagnetic four-potential $A^{\mu}$ as $F^{\mu \nu} \equiv \partial^{\mu} A^{\nu}-\partial^{\nu} A^{\mu}$ and the covariant derivative equals $D^{\mu} \equiv \partial^{\mu}+i e A^{\mu} ; \Lambda$ is the Majorana bispinor photino field, $\Psi$ is the Dirac bispinor electron field, $\phi_{L}$ and $\phi_{R}$ are the scalar left selectron and right selectron fields; the projectors $P_{L}$ and $P_{R}$ are defined in a standard way $P_{L} \equiv \frac{1}{2}\left(1-\gamma_{5}\right)$ and $P_{R} \equiv \frac{1}{2}\left(1+\gamma_{5}\right)$. Since we are interested in ultrarelativistic plasmas, the mass terms are neglected in the lagrangian.

As seen in the lagrangian (1), there is a self-interaction of selectron field due to the terms $\left(\phi_{L}^{*} \phi_{L}\right)^{2},\left(\phi_{R}^{*} \phi_{R}\right)^{2}$ and $-2 \phi_{L}^{*} \phi_{L} \phi_{R}^{*} \phi_{R}$. There is also a four-boson coupling $\phi_{L, R}^{*} \phi_{L, R} A^{\mu} A_{\mu}$ of the selectron with the electromagnetic field. Such a contact interaction is qualitatively different than that caused by a massless particle exchange. The scattering cross section in the absence of other interactions is isotropic in the center-of-mass frame of colliding particles and the energy and momentum transfers are bigger than that in electromagnetic interactions. Therefore, one expects that consequently transport properties of supersymmetric $\mathcal{N}=1$ QED plasma differ from those of QED plasma of electrons, positrons and photons. To test this expectation, in the next section we compute the cross sections of binary processes which occur in $\mathcal{N}=1$ SUSY QED plasma in the lowest non-trivial order of the coupling constant $\alpha \equiv e^{2} / 4 \pi$. Further on, the cross sections are used to derive the energy loss and momentum broadening of an energetic particle traversing the plasma.

\section{CROSS SECTIONS OF BINARY INTERACTIONS}

We discuss here the cross sections of all binary interactions contributing at the order of $\alpha^{2}\left(\alpha \equiv e^{2} / 4 \pi\right)$. The processes along with corresponding Feynman diagrams and cross sections are listed in Table I] Electrons, selectrons, photons and photinos are denoted as $e, \tilde{e}, \gamma, \tilde{\gamma}$. In every process the initial and/or final state particles can carry positive or negative charge; selectrons can be additionally ' $R$ ' (right) or ' $L$ ' (left). For each listed reaction the Feynman diagrams from the third column correspond to the first combination of charges of interacting particles. For example, the Feynman diagrams of the process $e^{\mp} e^{\mp} \longrightarrow e^{\mp} e^{\mp}$ actually represent the scattering $e^{-} e^{-} \longrightarrow e^{-} e^{-}$. The solid, dashed, wavy and double-solid lines in the Feynman diagrams correspond to electrons, selectrons, photons and photinos, respectively.

The computed cross sections, which are averaged over initial polarizations of colliding particles and summed over polarizations of final state particles, are expressed through the Mandelstam invariants $s, t$ and $u$ defined in the standard way. For a process denoted as $a b \longrightarrow c d$, we have

$$
s \equiv\left(p+p_{1}\right)^{2}, \quad t \equiv\left(p-p^{\prime}\right)^{2}, \quad u \equiv\left(p-p_{1}^{\prime}\right)^{2},
$$

where $p, p_{1}, p^{\prime}, p_{1}^{\prime}$ are four-momenta of particles $a, b, c, d$, respectively. The corresponding Feynman diagrams are drawn in such a way that particle $a$ comes from the upper left corner of the diagram, particle $b$ comes from the lower left corner, particle $c$ goes to the upper right, and particle $d$ goes to the lower right corner.

The first five processes from Table I occur in a supersymmetric QED plasma and in an electromagnetic plasma of electrons, positrons and photons. The remaining process are characteristic for the $\mathcal{N}=1$ SUSY QED plasma. As can be seen from Table I, the processes No. $6-8$ and $23-26$ are independent of momentum transfer. As a result, the scattering is isotropic in the center-of-mass frame of colliding particles. Such processes are qualitatively different from those in an electromagnetic plasma (processes No. $1-5$ ) which are dominated by an interaction with small momentum transfer. We also observe that for each plasma particle $e, \gamma, \tilde{e}, \tilde{\gamma}$ there is a process in which the cross section is independent of momentum transfer. 
TABLE I: Cross sections of binary processes

\begin{tabular}{|c|c|c|c|}
\hline$n^{0}$ & process & diagrams & cross section $\frac{d \sigma}{d t}$ \\
\hline 1 & $e^{\mp} e^{\mp} \longrightarrow e^{\mp} e^{\mp}$ & & $\frac{2 \pi \alpha^{2}}{s^{2}}\left(\frac{s^{2}+u^{2}}{t^{2}}+\frac{s^{2}+t^{2}}{u^{2}}+\frac{2 s^{2}}{t u}\right)$ \\
\hline 2 & $e^{ \pm} e^{\mp} \longrightarrow e^{ \pm} e^{\mp}$ & & $\frac{2 \pi \alpha^{2}}{s^{2}}\left(\frac{s^{2}+u^{2}}{t^{2}}+\frac{u^{2}+t^{2}}{s^{2}}+\frac{2 u^{2}}{t s}\right)$ \\
\hline 3 & $\gamma e^{\mp} \longrightarrow \gamma e^{\mp}$ & & $-\frac{2 \pi \alpha^{2}}{s^{2}}\left(\frac{s}{u}+\frac{u}{s}\right)$ \\
\hline 4 & $e^{ \pm} e^{\mp} \longrightarrow \gamma \gamma$ & & $\frac{2 \pi \alpha^{2}}{s^{2}}\left(\frac{t}{u}+\frac{u}{t}\right)$ \\
\hline 5 & $\gamma \gamma \longrightarrow e^{\mp} e^{ \pm}$ & & $\frac{2 \pi \alpha^{2}}{s^{2}}\left(\frac{t}{u}+\frac{u}{t}\right)$ \\
\hline 6 & $\tilde{\gamma} e^{\mp} \longrightarrow \tilde{\gamma} e^{\mp}$ & & $\frac{4 \pi \alpha^{2}}{s^{2}}$ \\
\hline 7 & $e^{ \pm} e^{\mp} \longrightarrow \tilde{\gamma} \tilde{\gamma}$ & & $\frac{4 \pi \alpha^{2}}{s^{2}}$ \\
\hline 8 & $\tilde{\gamma} \tilde{\gamma} \longrightarrow e^{\mp} e^{ \pm}$ & & $\frac{4 \pi \alpha^{2}}{s^{2}}$ \\
\hline 9 & $\tilde{\gamma} e^{\mp} \longrightarrow \gamma \tilde{e}_{L, R}^{\mp}$ & & $\frac{\pi \alpha^{2}}{s^{2}} \frac{t}{u}$ \\
\hline 10 & $\gamma \tilde{e}_{L, R}^{\mp} \longrightarrow \tilde{\gamma} e^{\mp}$ & & $\frac{2 \pi \alpha^{2}}{s^{2}} \frac{t}{u}$ \\
\hline 11 & $\gamma e^{\mp} \longrightarrow \tilde{\gamma} \tilde{e}_{L, R}^{\mp}$ & & $-\frac{2 \pi \alpha^{2}}{s^{2}} \frac{t}{s}$ \\
\hline 12 & $\tilde{\gamma} \tilde{e}_{L, R}^{\mp} \longrightarrow \gamma e^{\mp}$ & & $-\frac{2 \pi \alpha^{2}}{s^{2}} \frac{t}{s}$ \\
\hline 13 & $\tilde{e}_{L, R}^{ \pm} e^{\mp} \longrightarrow \tilde{\gamma} \gamma$ & & $-\frac{2 \pi \alpha^{2}}{s^{2}} \frac{s}{t}$ \\
\hline 14 & $\tilde{\gamma} \gamma \longrightarrow \tilde{e}_{L, R}^{\mp} e^{ \pm}$ & & $-\frac{\pi \alpha^{2}}{s^{2}} \frac{s}{t}$ \\
\hline
\end{tabular}


15

16

17

18

19

20

21

22

23

24

25

26

27

28
$\tilde{e}_{L, R}^{\mp} e^{\mp} \longrightarrow \tilde{e}_{L, R}^{\mp} e^{\mp}$

$\tilde{e}_{L, R}^{ \pm} e^{\mp} \longrightarrow \tilde{e}_{L, R}^{ \pm} e^{\mp}$

$e^{ \pm} e^{\mp} \longrightarrow \tilde{e}_{L, R}^{ \pm} \tilde{e}_{L, R}^{\mp}$

$\tilde{e}_{L, R}^{ \pm} \tilde{e}_{L, R}^{\mp} \longrightarrow e^{ \pm} e^{\mp}$

$\tilde{e}_{L, R}^{\mp} \tilde{e}_{L, R}^{\mp} \longrightarrow \tilde{e}_{L, R}^{\mp} \tilde{e}_{L, R}^{\mp}$

$\tilde{e}_{L, R}^{ \pm} \tilde{e}_{L, R}^{\mp} \longrightarrow \tilde{e}_{L, R}^{ \pm} \tilde{e}_{L, R}^{\mp}$

$\tilde{e}_{L, R}^{\mp} \tilde{e}_{R, L}^{\mp} \longrightarrow \tilde{e}_{L, R}^{\mp} \tilde{e}_{R, L}^{\mp}$

$\tilde{e}_{L, R}^{ \pm} \tilde{e}_{R, L}^{\mp} \longrightarrow \tilde{e}_{L, R}^{ \pm} \tilde{e}_{R, L}^{\mp}$

$\tilde{e}_{L, R}^{ \pm} \tilde{e}_{R, L}^{\mp} \longrightarrow \tilde{e}_{R, L}^{ \pm} \tilde{e}_{L, R}^{\mp}$

$\tilde{e}_{L, R}^{\mp} \tilde{e}_{L, R}^{\mp} \longrightarrow \tilde{e}_{R, L}^{\mp} \tilde{e}_{R, L}^{\mp}$

$\gamma \tilde{e}_{L, R}^{\mp} \longrightarrow \gamma \tilde{e}_{L, R}^{\mp}$

$\tilde{e}_{L, R}^{ \pm} \tilde{e}_{L, R}^{\mp} \longrightarrow \gamma \gamma$

$\gamma \gamma \longrightarrow \tilde{e}_{L, R}^{\mp} \tilde{e}_{L, R}^{ \pm}$

$\tilde{\gamma} \tilde{e}_{L, R}^{\mp} \longrightarrow \tilde{\gamma} \tilde{e}_{L, R}^{\mp}$
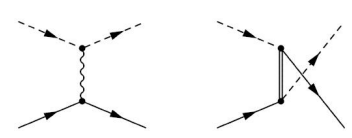

$-\frac{2 \pi \alpha^{2}}{s^{2}} \frac{s\left(s^{2}+u^{2}\right)}{u t^{2}}$

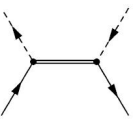

$-\frac{2 \pi \alpha^{2}}{s^{2}} \frac{u\left(s^{2}+u^{2}\right)}{s t^{2}}$

$\frac{\pi \alpha^{2}}{s^{2}} \frac{u\left(t^{2}+u^{2}\right)}{t s^{2}}$

$\frac{4 \pi \alpha^{2}}{s^{2}} \frac{u\left(t^{2}+u^{2}\right)}{t s^{2}}$
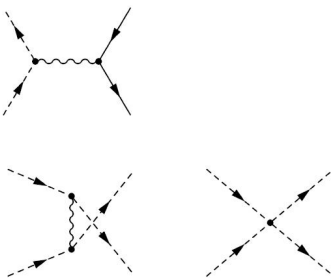

$\frac{4 \pi \alpha^{2}}{s^{2}}\left(\frac{u}{t}+\frac{t}{u}\right)^{2}$
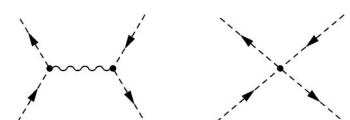

$\frac{4 \pi \alpha^{2}}{s^{2}}\left(\frac{s}{t}+\frac{t}{s}\right)^{2}$

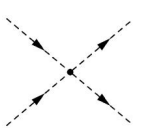

$\frac{4 \pi \alpha^{2}}{t^{2}}$

$\frac{4 \pi \alpha^{2}}{s^{2}} \frac{u^{2}}{t^{2}}$

$\frac{\pi \alpha^{2}}{s^{2}}$

$\frac{\pi \alpha^{2}}{s^{2}}$
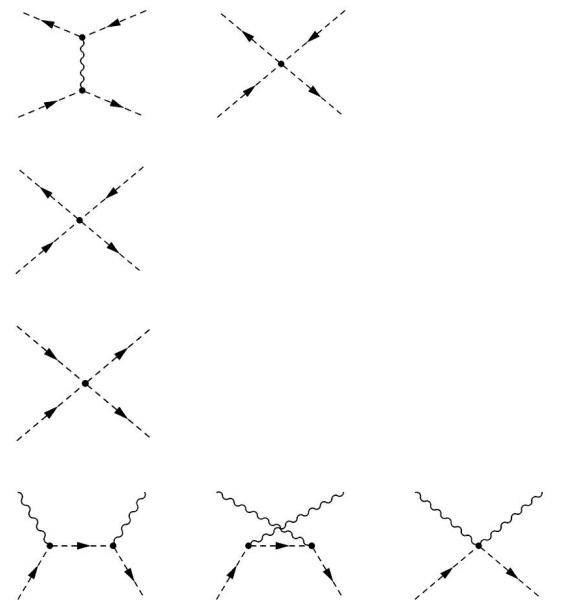

$\frac{4 \pi \alpha^{2}}{s^{2}}$
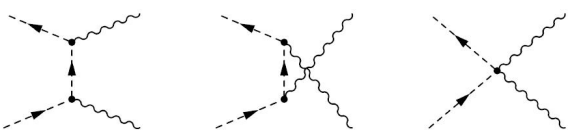

$\frac{8 \pi \alpha^{2}}{s^{2}}$
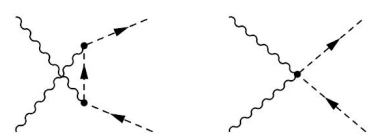

$\frac{2 \pi \alpha^{2}}{s^{2}}$

$-\frac{2 \pi \alpha^{2}}{s^{2}}\left(\frac{u}{s}+\frac{s}{u}\right)$ 
29

$$
\tilde{e}_{L, R}^{ \pm} \tilde{e}_{L, R}^{\mp} \longrightarrow \tilde{\gamma} \tilde{\gamma}
$$

30

$$
\tilde{\gamma} \tilde{\gamma} \longrightarrow \tilde{e}_{L, R}^{ \pm} \tilde{e}_{L, R}^{\mp}
$$

31

$$
\tilde{e}_{L, R}^{ \pm} e^{\mp} \longrightarrow e^{ \pm} \tilde{e}_{R, L}^{\mp}
$$

$$
e^{\mp} e^{\mp} \longrightarrow \tilde{e}_{L, R}^{\mp} \tilde{e}_{R, L}^{\mp}
$$

33

$$
\tilde{e}_{L, R}^{\mp} \tilde{e}_{R, L}^{\mp} \longrightarrow e^{\mp} e^{\mp}
$$
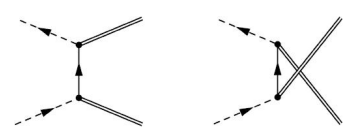

$\frac{4 \pi \alpha^{2}}{s^{2}}\left(\frac{u}{t}+\frac{t}{u}\right)$

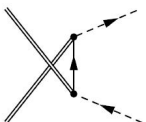

$\frac{\pi \alpha^{2}}{s^{2}}\left(\frac{u}{t}+\frac{t}{u}\right)$

$-\frac{2 \pi \alpha^{2}}{s^{2}}\left(\frac{s}{t}+\frac{t}{s}\right)$

$\frac{\pi \alpha^{2}}{s^{2}}\left(\frac{u}{t}+\frac{t}{u}\right)$

$\frac{4 \pi \alpha^{2}}{s^{2}}\left(\frac{u}{t}+\frac{t}{u}\right)$

\section{ENERGY LOSS}

As already mentioned, for each type of plasma particle there is a binary process with a cross section independent of $t$ or $u$. The matrix element of such a process is simply a number. We compute here the energy loss caused by such an interaction. To be specific, we consider a high energy selectron traversing an equilibrium $\mathcal{N}=1 \mathrm{SUSY}$ QED plasma. The selectron interacts with plasma particles of all types but we take into account only scattering on photons. This is only for demonstration but in general all processes, which contribute additively to the energy loss, must be included.

The selectron initial four-momentum is denoted as $p^{\mu}=(E, \mathbf{p})$ with $E \equiv|\mathbf{p}|$ and that of a plasma photon as $p_{1}^{\mu}=\left(E_{1}, \mathbf{p}_{1}\right)$. The final four-momenta of the selectron and photon are, respectively, $p^{\prime \mu}=\left(E^{\prime}, \mathbf{p}^{\prime}\right)$ and $p_{1}^{\prime \mu}=\left(E_{1}^{\prime}, \mathbf{p}_{1}^{\prime}\right)$. The energy loss of the selectron per unit length is then

$$
\frac{d E}{d x}=-\int d \Gamma\left(E-E^{\prime}\right)
$$

where $d \Gamma$ is the interaction rate given as

$$
d \Gamma=|\mathcal{M}|^{2} \frac{n\left(\mathbf{p}_{1}\right)}{16 E E^{\prime} E_{1} E_{1}^{\prime}} \frac{d^{3} p_{1}}{(2 \pi)^{3}} \frac{d^{3} p^{\prime}}{(2 \pi)^{3}} \frac{d^{3} p_{1}^{\prime}}{(2 \pi)^{3}}(2 \pi)^{4} \delta^{(4)}\left(p+p_{1}-p^{\prime}-p_{1}^{\prime}\right) ;
$$

$n\left(\mathbf{p}_{1}\right)$ is the distribution function of plasma photons and $\mathcal{M}$ denotes the scattering amplitude. We have neglected here the quantum factor $n\left(\mathbf{p}_{1}^{\prime}\right)+1$ which is important when the momentum of final state photon is of order of plasma temperature. Since the scattering process under consideration leads to a sizable momentum transfer and we are mostly interested in energy loss of a highly energetic particle, the factor can be safely ignored.

When $\mathcal{M}$ describes a scattering driven by a one-photon exchange, the formula (3) with the rate (4) leads to an infinite result due to the long range nature of electromagnetic interaction. The problem is cured by including the effect of screening in a plasma medium. In the case of photon-selectron scattering the matrix element equals $|\mathcal{M}|^{2}=4 e^{4}$ and it does not need any modification to provide a finite energy loss.

Substituting the interaction rate $\sqrt{4}$ with $|\mathcal{M}|^{2}=4 e^{4}$ into Eq. (3) and performing the trivial integration over $\mathbf{p}_{1}^{\prime}$, we obtain

$$
\frac{d E}{d x}=-\frac{e^{4}}{4} \int \frac{d^{3} p_{1}}{(2 \pi)^{3}} \frac{d^{3} q}{(2 \pi)^{3}} \frac{n\left(\mathbf{p}_{1}\right)\left(E-E^{\prime}\right)}{E^{2} E_{1} E_{1}^{\prime} q} 2 \pi \delta(\cos \theta-\overline{\cos \theta}),
$$

where $\mathbf{q} \equiv \mathbf{p}^{\prime}-\mathbf{p}=\mathbf{p}_{1}-\mathbf{p}_{1}^{\prime}$ is the momentum transfer and $q \equiv|\mathbf{q}| ; \theta$ is the angle between the vectors $\mathbf{p}$ and $\mathbf{q}$ and $\overline{\cos \theta}$ is the solution of the energy conservation equation

$$
\overline{\cos \theta}=\frac{\left(E+E_{1}-E_{1}^{\prime}\right)^{2}-E^{2}-q^{2}}{2 q E}
$$


provided $-1 \leq \overline{\cos \theta} \leq 1$

Now we make use of the assumption that the plasma is in thermal equilibrium and therefore is isotropic. As a result, the momentum distribution of plasma photons depends on $\mathbf{p}_{1}$ only through $E_{1}$ and we write it as $n\left(E_{1}\right)$. Consequently, the energy loss is independent of the orientation of the momentum p. Therefore, following [13] we average the formula (5) over the orientation of $\mathbf{p}$ with respect to $\mathbf{q}$ and we get

$$
\frac{d E}{d x}=\int \frac{d \Omega}{4 \pi} \frac{d E}{d x}=-\frac{e^{4}}{2^{8} \pi^{5}} \int d^{3} p_{1} d^{3} q \frac{n\left(E_{1}\right)\left(E-E^{\prime}\right)}{E^{2} E_{1} E_{1}^{\prime} q} .
$$

We write down the integral over $\mathbf{q}$ in spherical coordinates where the axis $z$ is along the momentum $\mathbf{p}_{1}$. Then, the integral over orientation of $\mathbf{p}_{1}$ is trivial and one obtains

$$
\frac{d E}{d x}=-\frac{e^{4}}{2^{5} \pi^{3}} \int_{0}^{\infty} d E_{1} E_{1}^{2} \int_{q_{\min }}^{q_{\max }} d q q^{2} \int_{\left(\cos \theta_{1}\right)_{\min }}^{\left(\cos \theta_{1}\right)_{\max }} d\left(\cos \theta_{1}\right) \frac{n\left(E_{1}\right)\left(E-E^{\prime}\right)}{E^{2} E_{1} E_{1}^{\prime} q}
$$

where $\theta_{1}$ is the angle between the vectors $\mathbf{p}_{1}$ and $\mathbf{q}$. The integration limits must be chosen in such a way that the energy conservation is satisfied. Instead of $\cos \theta_{1}$ it appears more convenient to use the variable $\omega \equiv E-E^{\prime}=$ $\sqrt{E_{1}^{2}-2 E_{1} q \cos \theta_{1}+q^{2}}-E_{1}$. Then, the expression (8) can be written in the form

$$
\frac{d E}{d x}=-\frac{e^{4}}{2^{5} \pi^{3} E^{2}} \int_{0}^{\infty} d E_{1} n\left(E_{1}\right) \int_{q_{\min }}^{q_{\max }} d q \int_{\omega_{\min }}^{\omega_{\max }} d \omega \omega
$$

To find the integration limits we express $\overline{\cos \theta}$, which is given by Eq. (6), through the variable $\omega$ and we demand that $-1 \leq \overline{\cos \theta} \leq 1$ keeping in mind that $-E_{1} \leq \omega \leq E$. Then, a somewhat lengthy but elementary analysis leads to the expression

$$
\begin{aligned}
\frac{d E}{d x}=-\frac{e^{4}}{2^{5} \pi^{3} E^{2}} & \left\{\int_{0}^{E} \mathrm{~d} E_{1} n\left(E_{1}\right)\left[\int_{0}^{E_{1}} d q \int_{-q}^{q} d \omega \omega+\int_{E_{1}}^{E} d q \int_{q-2 E_{1}}^{q} d \omega \omega+\int_{E}^{E+E_{1}} d q \int_{q-2 E_{1}}^{2 E-q} d \omega \omega\right]\right. \\
& \left.+\int_{E}^{\infty} d E_{1} n\left(E_{1}\right)\left[\int_{0}^{E} d q \int_{-q}^{q} d \omega \omega+\int_{E}^{E_{1}} d q \int_{-q}^{2 E-q} d \omega \omega+\int_{E_{1}}^{E+E_{1}} d q \int_{q-2 E_{1}}^{2 E-q} d \omega \omega\right]\right\}
\end{aligned}
$$

which after performing simple integrations over $q$ and $\omega$ gives

$$
\frac{d E}{d x}=-\frac{e^{4}}{2^{5} \pi^{3} E} \int_{0}^{\infty} d E_{1} n\left(E_{1}\right)\left(E E_{1}-E_{1}^{2}\right)
$$

To check correctness of rather complicated integration domain in Eq. [11, one observes that the integral (10) becomes simple when $\omega \equiv E-E^{\prime}$ is replaced by unity. Then, the integral

$$
\int \frac{d^{3} p^{\prime}}{(2 \pi)^{3} 2 E^{\prime}} \frac{d^{3} p_{1}^{\prime}}{(2 \pi)^{3} 2 E_{1}^{\prime}}(2 \pi)^{4} \delta^{(4)}\left(p+p_{1}-p^{\prime}-p_{1}^{\prime}\right)=\frac{1}{8 \pi}
$$

is Lorentz invariant and it can be easily computed in the center-of-mass frame. We have reproduced this result in an arbitrary frame performing the integration over the domain in $q-\omega$ space shown in Eq. (10).

The energy distribution of photons in equilibrium plasma is of Bose-Einstein form

$$
n(E)=\frac{2}{e^{\frac{E}{T}}-1},
$$

where the factor of 2 takes into account two photon polarizations and $T$ is the plasma temperature. Substituting the distribution (13) into Eq. (11), one finds

$$
\frac{d E}{d x}=-\frac{e^{4}}{2^{5} 3 \pi} T^{2}\left[1-\frac{12 \zeta(3)}{\pi^{2}} \frac{T}{E}\right],
$$

where $\zeta(z)$ is the zeta Riemann function and $\zeta(3) \approx 1.202$.

In the limit $E \gg T$ we have the result

$$
\frac{d E}{d x}=-\frac{e^{4}}{2^{5} 3 \pi} T^{2}
$$


which should be confronted with the energy loss of an energetic muon in ultrarelativistic electromagnetic plasma of electrons, positrons and photons [13]

$$
\frac{d E}{d x}=-\frac{e^{4}}{48 \pi^{3}} T^{2}\left(\ln \frac{E}{e T}+2.031\right) .
$$

As seen, the formulas 15, 16 are similar to each other up the logarithm term which is discussed later on. The similarity is rather surprising if one realizes how different are the differential cross sections of interest. Let us discuss why it happens.

The energy loss can be estimated as [14] $\frac{d E}{d x} \sim\langle\Delta E\rangle / \lambda$, where $\langle\Delta E\rangle$ is the typical change of particle's energy in a single collision and $\lambda$ is the particle's mean free path given as $\lambda^{-1}=\rho \sigma$ with $\rho \sim T^{3}$ being the density of scatterers and $\sigma$ denoting the cross section. For the differential cross section $\frac{d \sigma}{d t} \sim e^{4} / s^{2}$, the total cross section is $\sigma \sim e^{4} / s$. When a highly energetic particle with energy $E$ scatters on massless plasma particle, $s \sim E T$ and consequently $\sigma \sim e^{4} /(E T)$. The inverse mean free path is thus estimated as $\lambda^{-1} \sim e^{4} T^{2} / E$. When the scattering process is independent of momentum transfer, $\langle\Delta E\rangle$ is of order $E$ and we finally find $-\frac{d E}{d x} \sim e^{4} T^{2}$. When compared to the case of Coulomb scattering, the energy transfer in a single collision is much bigger but the cross section is smaller in the same proportion. Consequently, the two interactions corresponding to very different differential cross sections lead to very similar energy losses. The authors of [15] arrived to the analogous conclusion discussing viscous corrections to the distribution function caused by the collisions driven by a one-gluon exchange or by a $\phi^{4}$ contact interaction.

The Coulomb energy-loss formula (16) differs from (15) by the logarithm term which comes from the integration over the momentum transfer from the minimal $\left(q_{\min }\right)$ to maximal $\left(q_{\max }\right)$ value. The latter one is of order of energy of the test particle $\left(q_{\max } \sim E\right)$. In vacuum $q_{\min }=0$ and consequently the integral, which equals $\ln \left(q_{\max } / q_{\min }\right)$, diverges. In a plasma medium the long range Coulomb forces are screened and $q_{\min }$ is of order of Debye mass which in ultrarelativistic plasma is roughly $e T$. Thus, the logarithm term gets the form as in Eq. (16).

\section{BROADENING OF TRANSVERSE MOMENTUM}

We consider here a second transport characteristic of an equilibrium $\mathcal{N}=1$ SUSY QED plasma which is the momentum broadening of an energetic selectron due to its interaction with plasma photons. The quantity, which is usually denoted as $\hat{q}$, determines the magnitude of radiative energy loss of a highly energetic particle in a plasma medium [11]. It is defined as

$$
\hat{q}=\int d \Gamma q_{T}^{2}
$$

where $d \Gamma$ is, as previously, the interaction rate and $q_{T}$ is the momentum transfer to the selectron which is perpendicular to the selectron initial momentum.

Since $\hat{q}$ is computed in exactly the same way as the energy loss, it can be obtained by replacing $E^{\prime}-E$ by $q_{T}^{2}$ in the formulas from the previous section. Then, instead of equation (11) one finds

$$
\hat{q}=\frac{e^{4}}{2^{4} 3 \pi^{3} E} \int_{0}^{\infty} d E_{1} n\left(E_{1}\right)\left[E E_{1}^{2}+\frac{2}{3} E_{1}^{3}\right] .
$$

With the momentum distribution of plasma photons of the Bose-Einstein form (13), Eq. (18) gives

$$
\hat{q}=\frac{e^{4}}{12 \pi^{3}} T^{3}\left[\zeta(3)+\frac{\pi^{4}}{45} \frac{T}{E}\right] .
$$

When the momentum broadening is caused by scattering driven by one-photon exchange, $\hat{q}$ is of the order $e^{4} \ln (1 / e) T^{3}[16$. Therefore, we conclude that the momentum broadening and consequently the radiative energy loss of a highly energetic particle in the SUSY QED plasma is similar (up the logarithm term) to that in the electromagnetic plasma of electrons, positrons and photons. The logarithm occurs for the same reason as in the case of energy-loss formula.

\section{SUMMARY AND CONCLUSIONS}

After studying collective excitations of $\mathcal{N}=1$ SUSY QED plasma and finding them very similar to that of electromagnetic plasma of electrons, positrons and photons [1], we have focused our attention on collisional processes. First 
of all we have computed cross sections of all binary interactions which occur in the $\mathcal{N}=1$ SUSY QED plasma at the lowest nontrivial order of $\alpha$. We have found a class of processes, Compton scattering on selectrons for example, the cross sections of which are independent of momentum transfer. These processes, in contrast to those characteristic for QED plasma, are not dominated by interactions with small momentum transfer and there exists such a process for a plasma particles of every type. One can suspect that due to these processes, transport properties of a supersymmetric system are different than those of its non-supersymmetric counterpart. We have derived the formulas for energy loss and momentum broadening of an energetic particle, showing that the $\mathcal{N}=1$ SUSY QED plasma is actually surprisingly similar to the QED plasma.

\section{Acknowledgments}

We are very grateful to Mike Strickland for critical reading of the manuscript and various comments. This work was partially supported by the ESF Human Capital Operational Program under grant 6/1/8.2.1/POKL/2009 and by the Polish Ministry of Science and Higher Education under grants N N202 204638 and 667/N-CERN/2010/0.

[1] A. Czajka and St. Mrówczyński, Phys. Rev. D83, 045001 (2011).

[2] O. Aharony, S. S. Gubser, J. M. Maldacena, H. Ooguri, and Y. Oz, Phys. Rept. 323, 183 (2000).

[3] D. T. Son and A. O. Starinets, Ann. Rev. Nucl. Part. Sci. 57, 95 (2007).

[4] R. A. Janik, Lect. Notes Phys. 828, 147 (2011).

[5] S. Caron-Huot, P. Kovtun, G. D. Moore, A. Starinets, and L. G. Yaffe, JHEP 0612,015 (2006).

[6] S. C. Huot, S. Jeon, and G. D. Moore, Phys. Rev. Lett. 98, 172303 (2007).

[7] S. Caron-Huot and G. D. Moore, JHEP 0802, 081 (2008).

[8] J. P. Blaizot, E. Iancu, U. Kraemmer, and A. Rebhan, JHEP 0706, 035 (2007).

[9] P. M. Chesler and A. Vuorinen, JHEP 0611, 037 (2006).

[10] P. M. Chesler, A. Gynther, and A. Vuorinen, JHEP 0909, 003 (2009).

[11] R. Baier, Y. L. Dokshitzer, A. H. Mueller, S. Peigne, and D. Schiff, Nucl. Phys. B 484, 265 (1997).

[12] T. Binoth, E. W. N. Glover, P. Marquard, and J. J. van der Bij, JHEP 0205, 060 (2002).

[13] E. Braaten and M. H. Thoma, Phys. Rev. D44, 1298 (1991).

[14] S. Peigne and A. V. Smilga, Phys. Usp. 52, 659 (2009).

[15] K. Dusling, G. D. Moore, and D. Teaney, Phys. Rev. C 81, 034907 (2010).

[16] P. B. Arnold and W. Xiao, Phys. Rev. D78, 125008 (2008). 